ÉTUDES

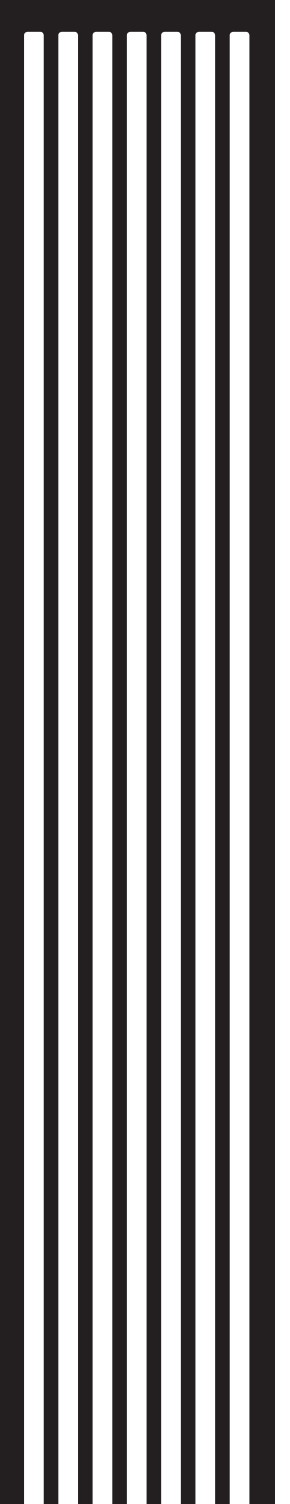





\title{
Análisis prosódico de la hesitación en los dialectos septentrionales y meridionales del español
}

\section{Prosodic Analysis of Hesitation Phenomena in the Northern and Southern Dialects of Spanish}

\author{
Kata Baditzné PÁlvölgyi [bpkat79@gmail.com] \\ Eötvös Loránd Tudományegyetem, Hungría
}

\section{RESUMEN}

El presente estudio se enfoca en los patrones de hesitación en las variedades septentrionales y meridionales del español peninsular. Se ha elaborado un corpus de 200 enunciados espontáneos con 100 enunciados procedentes de ambos territorios. El análisis sigue el protocolo ofrecido por Cantero (2019), según el cual para cada sílaba se representan los valores relevantes de entonación (en hercios), duración (en segundos) e intensidad (en decibelios), y, después, tales valores son estandarizados, para poder compararlos objetivamente. Según los resultados de la comparación, los dos corpus no presentan diferencias significativas en ninguno de los tres aspectos prosódicos expuestos. También se desprende de nuestros datos que los alargamientos y las pausas llenas sin función comunicativa específica no son prosódicamente prominentes respecto a su contexto, para garantizar que el hablante mantenga el turno conversacional ininterrumpido.

\section{Palabras clave}

Hesitación; pausas llenas; alargamientos; prosodia; estandarización

\begin{abstract}
This research focuses on the prosodic patterns of hesitations attested in northern and southern dialects of European Spanish. A corpus of 200 spontaneous utterances has been compiled (including 100 utterances from both dialects). The analysis has been carried out following the protocol offered by Cantero (2019), in which the representative values of intonation (in $\mathrm{Hz}$ ), duration (in sec) and intensity (in $\mathrm{dB}$ ) are taken for each syllable, and then these values undergo a process of standardization, in order to be comparable objectively. According to the results of the comparison, the two corpora did not present significant differences in any of the three exposed prosodic aspects. It also appears from our data that lengthenings and filled pauses without specific communicative function are not prosodically prominent with respect to their context, to ensure that the speaker keeps the conversational turn uninterrupted.
\end{abstract}




\section{KEYWORDS}

Hesitation; filled pauses; lengthenings; prosody; standardization

RECIBIDO 2020-03-22; ACEPTADO 2020-06-10

El presente estudio se ha realizado gracias al apoyo del "Nuevo Programa Nacional de Excelencia" ÚNKP19-4 del Ministerio de Innovación y Tecnología de Hungría y de la beca de investigación "János Bolyai” de la Academia Húngara de Ciencias.

\section{Introducción}

La hesitación se considera un fenómeno de disfluencia. Existen varias subcategorizaciones relacionadas con tales fenómenos (Eklund 2004; Lickley 1994, 2018; Neuberger 2014; Rordíguez et al. 2001; Shriberg 1994), pero en esta investigación se eligió la de Gósy (2002), que diferencia entre las disfluencias que se presentan debido a la realización errónea y las que surgen debido a la inseguridad del hablante. Los fenómenos relacionados con este último grupo incluyen: repeticiones, falsas partidas, pausas silenciosas, pausas sonoras y alargamientos. La presente investigación se centra en estos dos últimos fenómenos de disfluencia, ya que son los dos tipos más frecuentes de hesitación (Deme y Markó 2013).

Las prolongaciones se aplican para ganar tiempo sin implicar necesariamente la interrupción de la elocución. Su objetivo es reducir la velocidad del habla sin afectar la comunicación (Rebollo Couto 1997: 667). Las pausas llenas carecen de un significado concreto y se emplean para proporcionar al hablante tiempo para planificar y reorganizar su discurso (Stepanova 2007, citado por Machuca y Ríos 2016). Las pausas llenas y los alargamientos pueden considerarse dos fenómenos diferentes de disfluencia acústica (Rodríguez et al. 2001, 2015), pero el presente estudio se basa en autores que sostienen que las prolongaciones se subsumen bajo las pausas llenas (Campione y Véronis 2005; Maclay y Osgood 1959, citado por Machuca 2018), como un tipo especial "léxico" (Blondet 2001, Villa et al. 2017), y los dos fenómenos se tratarán juntos, centrándonos en el aspecto prosódico de las prolongaciones y pausas llenas en dos dialectos bien definidos del español europeo.

En la Península Ibérica podemos distinguir dos áreas dialectales principales que comparten varias características en su pronunciación: las variedades del norte (incluidas también las centrales) y los dialectos del sur (incluidas las variedades habladas en las Islas Canarias; Hualde 2014: 285-288). Teniendo en cuenta esta dicotomía, el objetivo de este estudio ha sido comparar las estrategias de hesitación aplicadas por los dialectos del español del norte y del sur, en tres vertientes relacionadas con la prosodia: (a) la entonación, (b) la intensidad y (c) la duración de los segmentos alargados y de las pausas llenas. Según nuestras hipótesis:

1) no se darán diferencias considerables entre los primeros dos aspectos (entonación e intensidad), pero sí en la duración. Esta divergencia se deberá a que los dialectos meridionales, cuya articulación generalmente se considera más rápida debido a la elisión de ciertos sonidos (como la -s final de sílaba o la - $d$ - intervocálica), tienden, en efecto, a realizar los segmentos más rápidos (Toledo 2010). Esto causará que las prolongaciones y las pausas llenas, de la misma duración absoluta que en los dialectos septentrionales, relativamente se realicen más largas. 
2) los segmentos alargados y las pausas llenas sin función comunicativa específica (salvo la de mantener el turno de palabra) no presentarán rasgos prosódicos de melodía e intensidad que destaquen respecto a su contexto. La explicación de tal fenómeno residirá en que este tipo de alargamientos y de pausas sonoras sirven, como se ha dicho, para mantener el turno de palabra (Maclay y Osgood 1959, citado por de Leeuv 2007). Si estos fenómenos de disfluencia no presentan variaciones muy notorias en el habla, no entrecortan el discurso, y aparentemente podrán garantizar la fluidez.

Para validar las hipótesis, complementando los resultados parciales de Baditzné (2020), realizamos una investigación que contrastó 100 enunciados emitidos por hablantes de dialectos del español del norte con 100 enunciados procedentes de informantes del sur de España. En ambos corpus aplicamos la metodología propuesta por Cantero (2019), el análisis prosódico trifásico del habla, para poder comparar los datos objetivamente.

\section{El corpus utilizado}

El corpus se obtuvo, por una parte, mediante las actividades de 'Map Task' en el Atlas Interactivo de Entonación Románica compilado por Prieto et al. (2010-2014) y por otra, de entrevistas en YouTube. De esta forma conseguimos solo muestras de habla espontánea. Se seleccionaron 32 hablantes, 16 informantes del norte ( 8 hombres y 8 mujeres) y 16 del sur de España ( 8 hombres y 8 mujeres), procedentes de grabaciones de 4 horas 51 minutos y 30 segundos en total. En el corpus septentrional, se han detectado 147 fenómenos de hesitación, en comparación con 137 casos en el corpus meridional. El siguiente mapa muestra la procedencia de los informantes (Fig. 1).
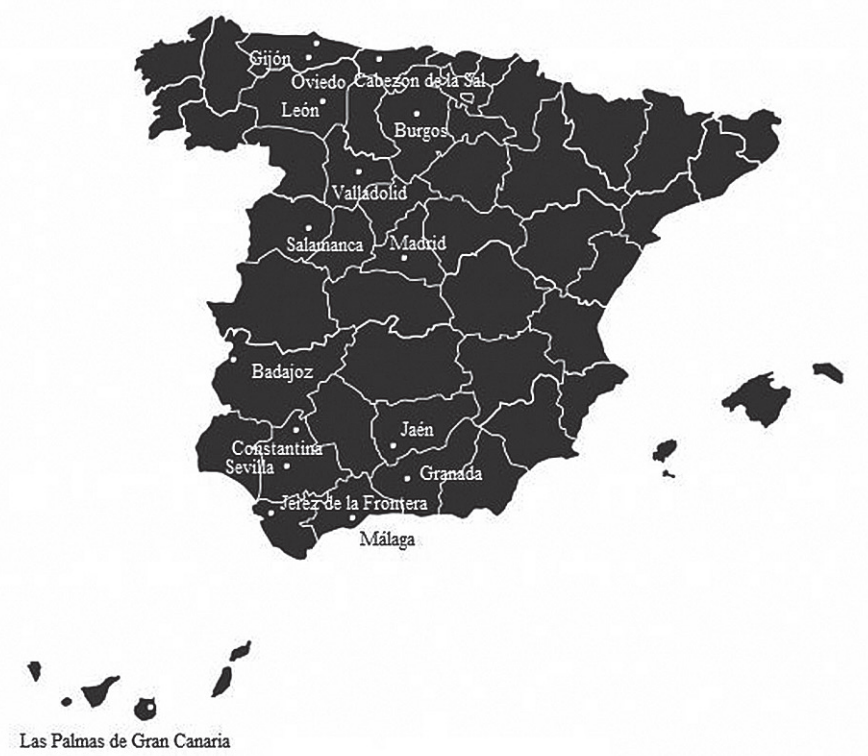

Figura 1. Procedencia de los informantes. 
Como se desprende del mapa, no hemos recogido enunciados de ningún territorio bilingüe donde otra lengua pudiera tener influencia sobre el español; no se incluyó, por esta razón, ninguna comunidad que fuera parcialmente catalanoparlante (Cataluña, Valencia o las Islas Baleares); ni el País Vasco, la Rioja (por el vasco) o Galicia (por la presencia del gallego).

La siguiente tabla muestra los datos relacionados de los informantes.

\begin{tabular}{|c|c|c|c|c|c|c|c|}
\hline 离 & 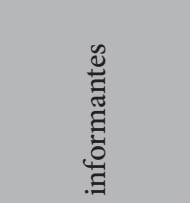 & 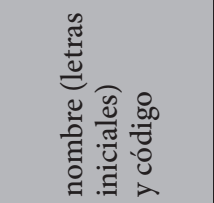 & 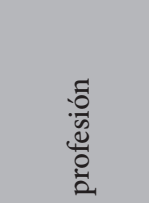 & 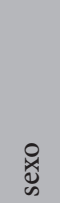 & 宽 & 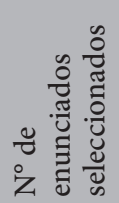 & 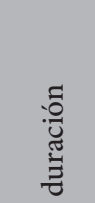 \\
\hline \multirow{2}{*}{$\begin{array}{l}\text { Gijón } \\
\text { (Map Task) }\end{array}$} & Informante 1 & GP; EGI-1 & estudiante & $\mathrm{f}$ & 24 & 5 & \multirow{2}{*}{$5: 49^{\prime}$} \\
\hline & Informante 2 & AL; EGI-2 & estudiante & $\mathrm{f}$ & 22 & 7 & \\
\hline \multirow{2}{*}{\begin{tabular}{|l} 
Oviedo \\
(Map Task)
\end{tabular}} & Informante 1 & GP; EOV-1 & estudiante & $\mathrm{f}$ & 20 & 9 & \multirow{2}{*}{$4: 52^{\prime}$} \\
\hline & Informante 2 & MR; EOV-2 & estudiante & $\mathrm{f}$ & 25 & 6 & \\
\hline \multirow{2}{*}{$\begin{array}{l}\text { Cabezón de la Sal } \\
\text { (Map Task) }\end{array}$} & Informante 1 & NEO; ECA-1 & profesora & $\mathrm{f}$ & 31 & 12 & \multirow{2}{*}{ 11:50' } \\
\hline & Informante 2 & AOZ; ECA-2 & profesora & $\mathrm{f}$ & 31 & 2 & \\
\hline \multirow{2}{*}{$\begin{array}{l}\text { Madrid } \\
\text { (Map Task) }\end{array}$} & Informante 1 & CTN; EMA-1 & ninguna & $\mathrm{f}$ & 33 & 3 & \multirow{2}{*}{$14: 00$} \\
\hline & Informante 2 & SBL; EMA-2 & ninguna & $\mathrm{f}$ & 37 & 5 & \\
\hline \multirow{2}{*}{$\begin{array}{l}\text { Salamanca } \\
\text { (entrevistas) }\end{array}$} & Informante 1 & FI; ESA-1 & político & $\mathrm{m}$ & 49 & 7 & $4: 39^{\prime}$ \\
\hline & Informante 2 & CG; ESA-2 & político & $\mathrm{m}$ & 57 & 6 & $21: 36^{\prime}$ \\
\hline \multirow{2}{*}{$\begin{array}{l}\text { Burgos } \\
\text { (entrevistas) }\end{array}$} & Informante 1 & JV; EBU-1 & político & $\mathrm{m}$ & 59 & 6 & 15:53' \\
\hline & Informante 2 & LT; EBU-2 & político & $\mathrm{m}$ & 36 & 6 & $16: 16$ \\
\hline \multirow{2}{*}{\begin{tabular}{|l} 
Ávila \\
(entrevistas)
\end{tabular}} & Informante 1 & ÁA; EAV-1 & político & $\mathrm{m}$ & 60 & 6 & 22:31' \\
\hline & Informante 2 & MÁGN; EAV-2 & político & $\mathrm{m}$ & 59 & 7 & $25: 23^{\prime}$ \\
\hline \multirow{2}{*}{$\begin{array}{l}\text { León } \\
\text { (entrevistas) }\end{array}$} & Informante 1 & AS; ELE-1 & político & $\mathrm{m}$ & 56 & 6 & $26: 30^{\prime}$ \\
\hline & Informante 2 & JADD; ELE-2 & político & $\mathrm{m}$ & 51 & 6 & $27: 24^{\prime}$ \\
\hline edad (años; media) & \multicolumn{7}{|c|}{40,63} \\
\hline enunciados (total) & \multicolumn{7}{|c|}{100} \\
\hline
\end{tabular}

\begin{tabular}{|c|c|c|c|c|c|c|c|}
\hline 范 & 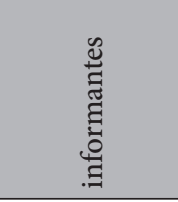 & 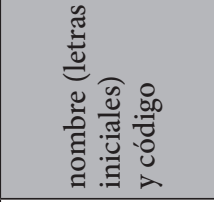 & 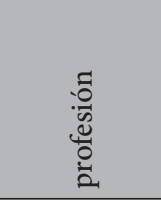 & dù & तु & 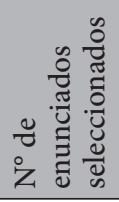 & 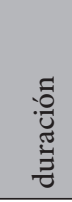 \\
\hline \multirow{2}{*}{$\begin{array}{l}\text { Islas Canarias } \\
\text { (Map Task) }\end{array}$} & Informante 1 & AAG; ECAN-1 & profesora & $\mathrm{f}$ & 38 & 6 & \multirow{2}{*}{$4: 41^{\prime}$} \\
\hline & Informante 2 & AAH; ECAN-2 & profesor & $\mathrm{m}$ & 38 & 8 & \\
\hline \multirow{2}{*}{$\begin{array}{l}\text { Jaén } \\
\text { (Map Task) }\end{array}$} & Informante 1 & SB; EJA-1 & estudiante & $\mathrm{f}$ & 22 & 10 & \multirow{2}{*}{$4: 14^{\prime}$} \\
\hline & Informante 2 & DG; EJA-2 & estudiante & $\mathrm{m}$ & 21 & 1 & \\
\hline \multirow{2}{*}{$\begin{array}{l}\text { Constantina } \\
\text { (Map Task) }\end{array}$} & Informante 1 & LSN; ECO-1 & estudiante & $\mathrm{f}$ & 23 & 8 & \multirow{2}{*}{$2: 42^{\prime}$} \\
\hline & Informante 2 & MSC; ECO-2 & estudiante & $\mathrm{f}$ & 22 & 8 & \\
\hline \multirow{2}{*}{$\begin{array}{l}\text { Jerez de la Frontera } \\
\text { (Map Task) }\end{array}$} & Informante 1 & MGA; EJE-1 & empresaria & $\mathrm{f}$ & 41 & 3 & \multirow{2}{*}{$3: 15^{\prime}$} \\
\hline & Informante 2 & PM; EJE-2 & empresario & $\mathrm{m}$ & 46 & 6 & \\
\hline
\end{tabular}




\begin{tabular}{|c|c|c|c|c|c|c|c|}
\hline 营 & 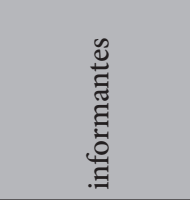 & 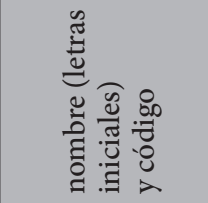 & 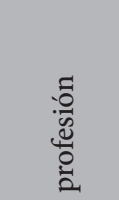 & 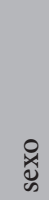 & Tु & 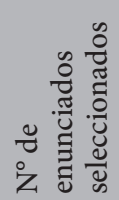 & 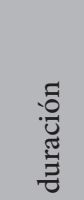 \\
\hline \multirow{2}{*}{$\begin{array}{l}\text { Málaga } \\
\text { (entrevistas) }\end{array}$} & Informante 1 & MÁJ; EMAL-1 & político & $\mathrm{m}$ & 49 & 6 & 14:05' \\
\hline & Informante 2 & EB; EMAL-2 & político & $\mathrm{m}$ & 45 & 6 & 18:14' \\
\hline \multirow{2}{*}{$\begin{array}{l}\text { Sevilla } \\
\text { (entrevistas) }\end{array}$} & Informante 1 & PP; ESE-1 & política & $\mathrm{f}$ & 50 & 7 & \multirow{2}{*}{$15: 19$} \\
\hline & Informante 2 & JE; ESE-2 & político & $\mathrm{m}$ & 51 & 6 & \\
\hline \multirow{2}{*}{$\begin{array}{l}\begin{array}{l}\text { Badajoz } \\
\text { (entrevistas) }\end{array} \\
\end{array}$} & Informante 1 & FJF; EBA-1 & político & $\mathrm{m}$ & 47 & 6 & $12: 18^{\prime}$ \\
\hline & Informante 2 & GFV; EBA-2 & político & $\mathrm{m}$ & 59 & 6 & $6: 20^{\prime}$ \\
\hline \multirow{2}{*}{$\begin{array}{l}\begin{array}{l}\text { Granada } \\
\text { (entrevistas) }\end{array} \\
\end{array}$} & Informante 1 & MJLG; EGR-1 & política & $\mathrm{f}$ & 55 & 6 & $8: 37^{\prime}$ \\
\hline & Informante 2 & LGCH; EGR-2 & política & $\mathrm{f}$ & 47 & 7 & $5: 03^{\prime}$ \\
\hline edad (años; media) & \multicolumn{7}{|c|}{40,88} \\
\hline enunciados (total) & \multicolumn{7}{|c|}{100} \\
\hline
\end{tabular}

Tabla 1. Datos relacionados con los informantes.

\section{Metodología}

La base teórica utilizada en este trabajo se basa en el protocolo para el Análisis Prosódico del Habla (abreviado como PAS, Cantero 2019). El tono, la duración y la intensidad se consideran características suprasegmentales y, por esta razón, son relativamente difíciles de interpretar. Primero, porque debemos ignorar las características dependientes del hablante que no tienen significado lingüístico, y segundo, porque las unidades prosódicas tienen una importancia relativa solo con respecto a las unidades adyacentes, por lo que carecen de información por sí solas.

El Análisis Melódico del Habla (MAS) (Cantero y Font-Rotchés 2009) y su última implementación de la teoría, Análisis Prosódico del Habla (PAS) (2019) ofrecen una solución para superar estas dificultades. En cuanto al análisis, la primera fase es acústica, asistida por un software de análisis acústico como Praat (Boersma y Weenink 2019). El segundo paso es la representación prosódica: para concentrarse solo en las características prosódicamente relevantes, es necesario ignorar las variaciones irrelevantes y reducir los datos en el caso de cada sílaba a un valor prosódico característico (en el caso de entonación, en Hz; en el caso de intensidad, en dB, y en el caso de duración, en segundos). El tercer paso es la estandarización de los datos: los gráficos de datos prosódicos se representan teniendo en cuenta no los valores absolutos, sino los relativos, ya que cada sílaba recibe un porcentaje basado en su ascenso / descenso prosódico experimentado con respecto a la sílaba anterior.

La estandarización de los datos prosódicos no es una idea reciente en la investigación lingüística. La estandarización de los contornos melódicos se realizó primero usando semitonos de la "Escuela Holandesa", también conocida como el modelo IPO. El trabajo más emblemático de este enfoque es t'Hart et al. (1990), seguido más tarde por varias investigaciones en diferentes idiomas (Adriaens 1991, Beaugendre 1994, Odé y van Heuven 1994). En español, Garrido $(1991,1996)$ y 
Estruch et al. (1999) trabajaron con métodos similares de estilización automática (Baditzné 2012). La diferencia entre las curvas estandarizadas en el modelo MAS y las de la escuela holandesa estriba en que el modelo MAS utiliza porcentajes para los valores estándares, que es un sistema más fácil de manejar que el que emplea semitonos. El contorno estandarizado está representado por una línea que comienza en un valor arbitrario del 100\% y se ancla en cada sílaba, que se caracteriza por un porcentaje basado en su posición tonal en comparación con la sílaba anterior. Si la sílaba se encuentra más baja, es un porcentaje negativo, y si es más alta que la sílaba anterior, es positivo. Aunque el modelo MAS se aplicó por primera vez para el español (Cantero et al. 2005, Cantero y Font-Rotchés 2007; 2020, Font-Rotchés y Mateo 2011), también disponemos de varias investigaciones realizadas en este marco en otros idiomas, véanse por ejemplo el catalán (Font-Rotchés 2007, 2008, 2009) o el chino (Kao 2011). Para una aplicación parcial del español, véase también Patiño (2008). En húngaro, se realiza un análisis en parte similar en Olaszy y Koutny (2001), que también se vale de porcentajes y contornos estilizados.

Partiendo del modelo MAS, en Cantero (2019) el análisis se extendió a otros aspectos prosódicos, a la intensidad y a la duración también. El modelo extendido se denomina PAS (Prosodic Analysis of Speech), y en el presente estudio vamos a presentarlo más detalladamente, ya que sirvió como base de nuestro análisis para investigar en ambos corpus los siguientes aspectos: (a) la entonación, (b) la intensidad y (c) la duración de tanto los segmentos prolongados como de las pausas llenas.

\subsection{El aspecto melódico}

En el caso del aspecto entonativo, en la obtención de datos seguimos el proceso expuesto en Cantero (2019: 489-491). Los enunciados se visualizan mediante el software de análisis acústico Praat (Boersma y Weenink 2019), y después procedemos a la fase de conseguir los valores de frecuencia fundamental (f0) en el caso de cada sílaba.

Primero, nos libramos de las variaciones micromelódicas irrelevantes, reduciendo cada sílaba a un valor tonal característico. En el caso de inestabilidad tonal dentro de una sílaba, se toman los valores extremos de la f0. Después, cada valor absoluto (medido en hercios) se convierte en un valor relativo, dependiendo del valor directamente anterior: al primer valor del enunciado se le asigna un valor arbitrario '100', y los siguientes valores representan la distancia tonal medida en \% respecto a la sílaba anterior. Por ejemplo, un salto de $152 \mathrm{~Hz}$ a $170 \mathrm{~Hz}$ en la siguiente sílaba resultaría en los valores de 100 y 112 respectivamente, ya que entre 152 y 170 hay un ascenso del 12\%.

A continuación, ejemplificamos el proceso más detalladamente con un enunciado de Badajoz, $Y$ viendo el patrimonio monumental de la... La siguiente imagen muestra la curva entonativa en gris (generado por Praat, el texto es adición nuestra). 


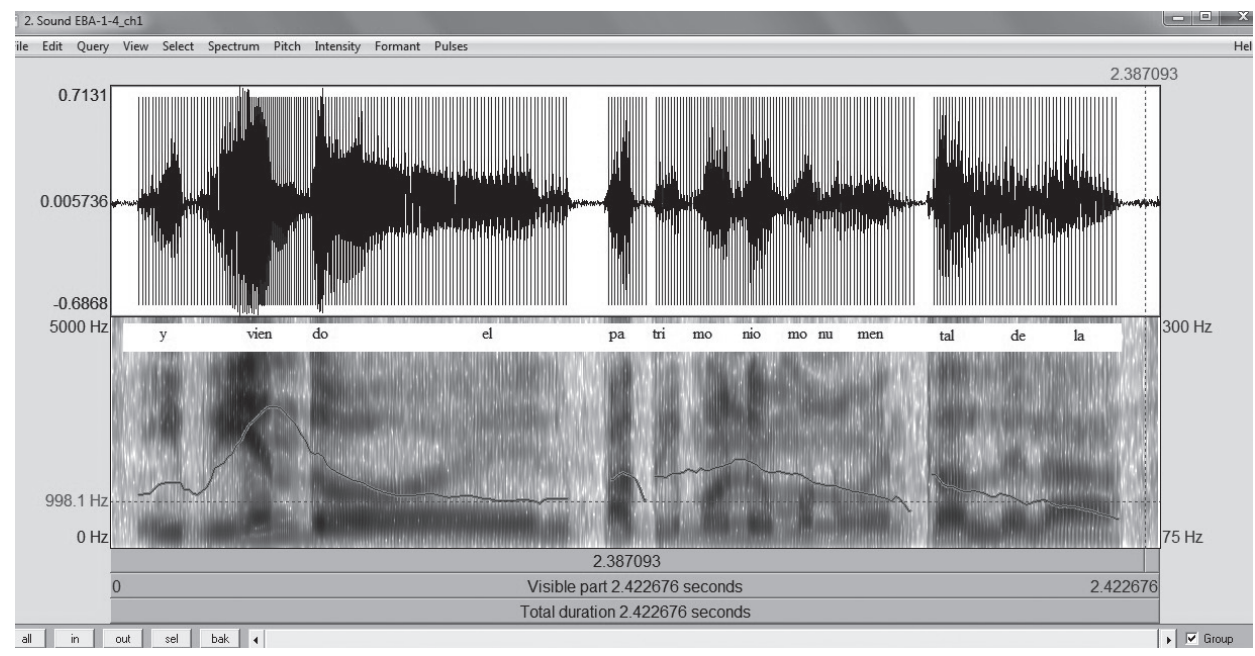

Figura 2. La curva melódica del enunciado Y viendo el patrimonio monumental de la..., de Badajoz.

Para reducir la curva entonativa a lo melódicamente relevante, nos libramos de las variaciones micromelódicas insignificantes y cada sílaba se reduce a un solo valor tonal si es melódicamente estable. Las vocales melódicamente estables se miden en su punto medio. En el caso de este enunciado, la primera vocal [i] de la palabra $y$ parece inestable, pero en realidad, el movimiento tonal dentro de esta sílaba es imperceptible al oído humano, ya que la diferencia tonal entre sus valores extremos $(125,5 \mathrm{~Hz}$ y $138,4 \mathrm{~Hz})$ no alcanza el umbral de percepción, que es, en el caso del español, del 10\% (Font-Rotchés y Mateo Ruiz 2011).

En el caso de la sílaba vien-, sin embargo, no podemos tomar el valor central de la vocal (que sería de solo $183 \mathrm{~Hz}$ ), porque dentro de la sílaba la melodía alcanza incluso los $212 \mathrm{~Hz}$ en su punto más alto, por lo tanto, es este el valor que deberíamos anotar en vez del valor central para no simplificar la melodía demasiado (véase Fig. 4).

Siguiendo este mecanismo, podemos obtener la curva melódica desprovista de las variaciones micromelódicas irrelevantes (Fig. 5). En el caso de la sílaba pa-, como tenemos una inestabilidad tonal superior a $10 \%$, se toman los dos valores extremos (en la representación, el punto delante de la vocal marca esta inflexión tonal interna).

Después de esta fase, procedemos a la estandarización. Todos los valores absolutos (medidos en $\mathrm{Hz}$ ) se convierten en relativos, dependiendo siempre del valor anterior: al primer valor del enunciado se le asigna el valor arbitrario '100', y los valores siguientes representan la distancia tonal medida en \% respecto a la sílaba anterior. Por ejemplo, el salto de $138 \mathrm{~Hz}$ a $212 \mathrm{~Hz}$ en la sílaba siguiente resultaría en los valores de 100 y 154, respectivamente, ya que entre 138 y 212 hay un ascenso del 53,62\%. En la Figura 6 ilustramos cómo los valores absolutos en hercios (línea gris clara) se convierten en valores relativos (línea gris oscura). 

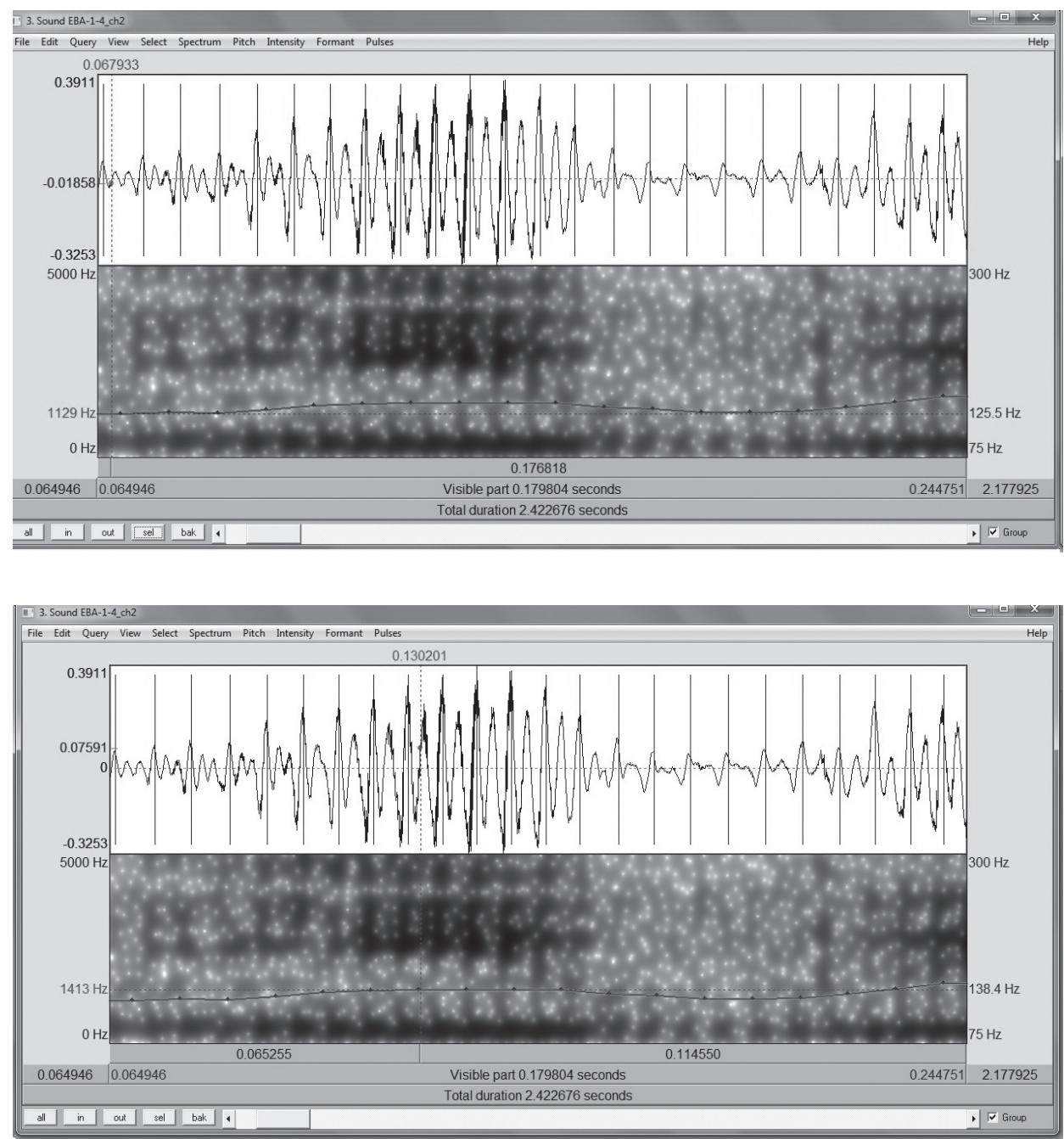

Figura 3. Imagen amplificada de la primera sílaba $y$.

La curva estandarizada garantiza de este modo que las melodías descritas sean objetivamente comparables entre sí, independientemente de las características tonales individuales de los locutores (p. ej. si se trata de un niño con una altura tonal mucho más alta que un hombre; lo que importaría son las proporciones de los movimientos tonales y no los valores absolutos de cada curva en sí). Las dos curvas son melódicamente idénticas, pero para validar si la copia estandarizada coincide melódicamente con la curva original, se pueden realizar pruebas perceptivas.

En nuestro análisis, solo comparamos dos datos en los dos corpus: el valor melódico estándar en el segmento afectado y el siguiente, es decir, la proporción del movimiento tonal en \% hacia el segmento afectado (sea alargado o una pausa llena), y la proporción del movimiento tonal desde la sílaba afectada hasta la siguiente sílaba (indicados con flechas en la Figura 7). 


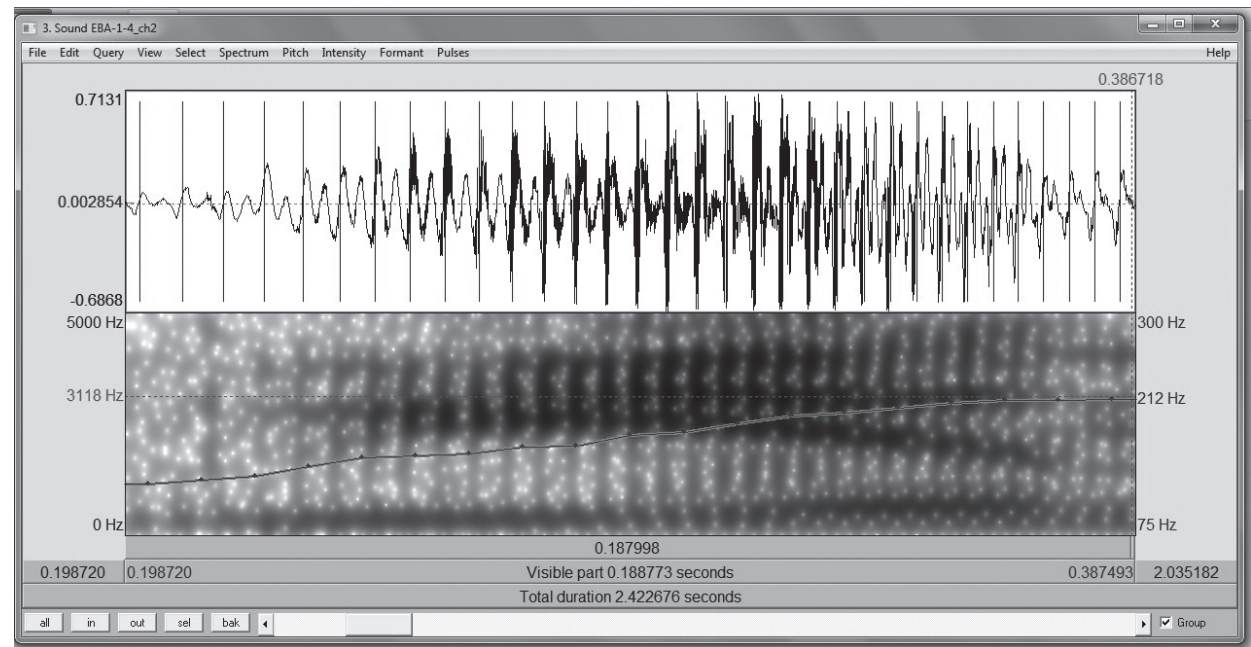

Figura 4. Imagen amplificada de la sílaba vien-.

EBA-1-4

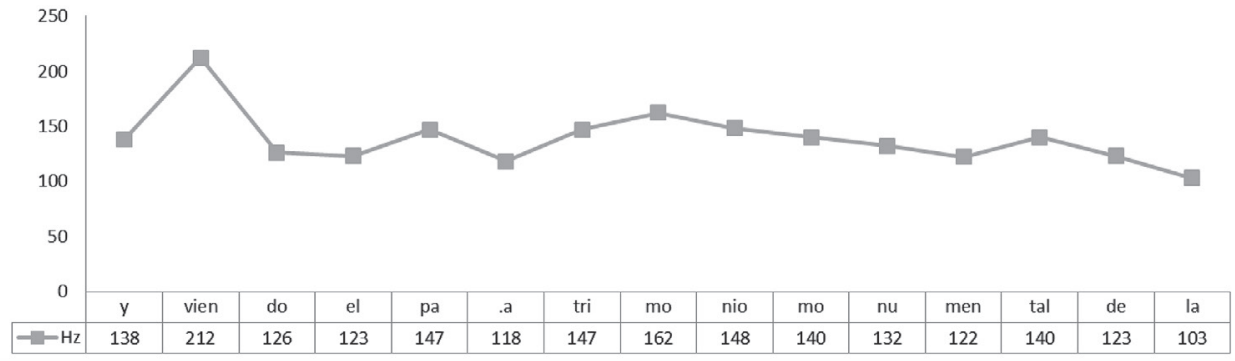

Figura 5. Curva tonal de valores absolutos del enunciado de Badajoz $Y$ viendo el patrimonio monumental de la...

EBA-1-4

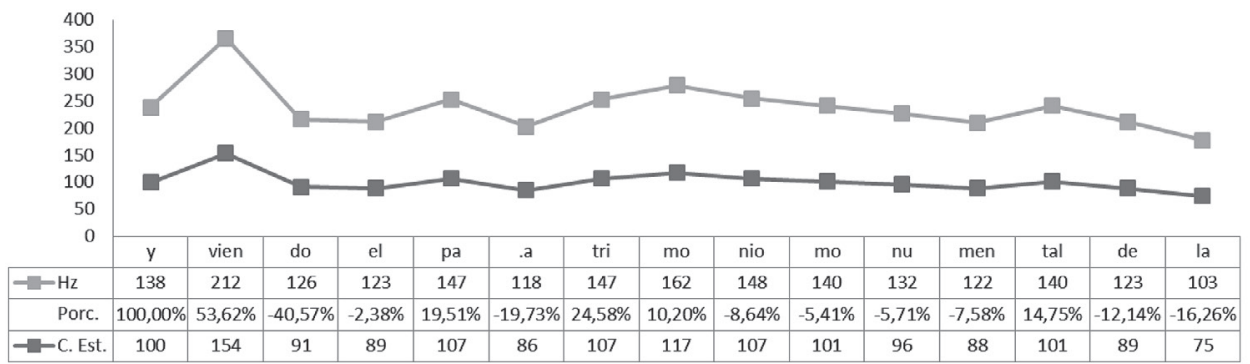

Figura 6. Curva de valores tonales absolutos (verde) y relativos (azul) del enunciado de Badajoz Y viendo el patrimonio monumental de la... 


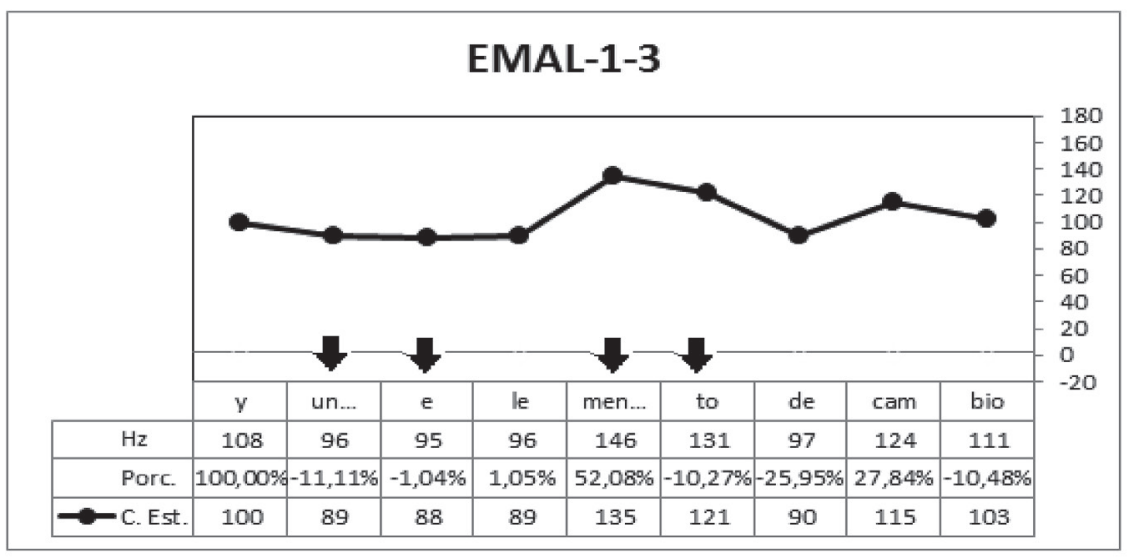

Figura 7. Gráfico de un enunciado malagueño (representación estandarizada de los datos de melodía).

\subsection{El aspecto dinámico}

La intensidad se mide en decibelios, en el caso de cada sílaba obtenemos los datos después de visualizar los archivos de sonido en Praat. A cada sílaba le corresponde un pico de intensidad, cuyo valor en $\mathrm{dB}$ servirá como punto de anclaje para la curva de intensidad (véase Figura 8).

Siguiendo el protocolo expuesto en Cantero (2019: 491-494), tras obtener los valores para cada sílaba, los estandarizamos siguiendo la pauta presentada ya en el caso del análisis melódico, y adquirimos una curva estandarizada de la intensidad. Paralelamente a lo que hicimos en el caso del análisis melódico, durante el análisis dinámico también comparamos dos valores: la proporción del movimiento dinámico en \% hasta el segmento afectado (prolongación o pausa sonora), y la proporción del movimiento dinámico desde esta sílaba hasta la siguiente (indicados con flechas en el gráfico).

\subsection{El aspecto rítmico}

En cuanto a la duración, seguimos el proceso de obtención de datos y estandarización partiendo del modelo ofrecido por Cantero (2019: 494-495). Cantero toma la distancia medida entre los dos primeros picos de intensidad del enunciado como valor de referencia, correspondientes a las dos primeras vocales nucleares, y las sílabas posteriores (cada una con un pico de intensidad) presentan valores proporcionales expresados en porcentajes con respecto a este primer valor de distancia. El algoritmo nos permite ver hasta qué punto una sílaba alargada es más larga que los otros intervalos silábicos dentro del mismo enunciado. En nuestro caso, hemos medido como primera distancia la duración entre el pico de intensidad de la primera sílaba del enunciado y el pico de intensidad de la segunda. La última distancia la obtuvimos desde el último pico de intensidad perteneciente a la última sílaba hasta el final del enunciado (véase Fig. 9).

Podría surgir la pregunta de por qué no medir las duraciones absolutas de cada sílaba, en vez de las distancias entre picos de intensidad. Un argumento a favor de medir las distancias entre picos 


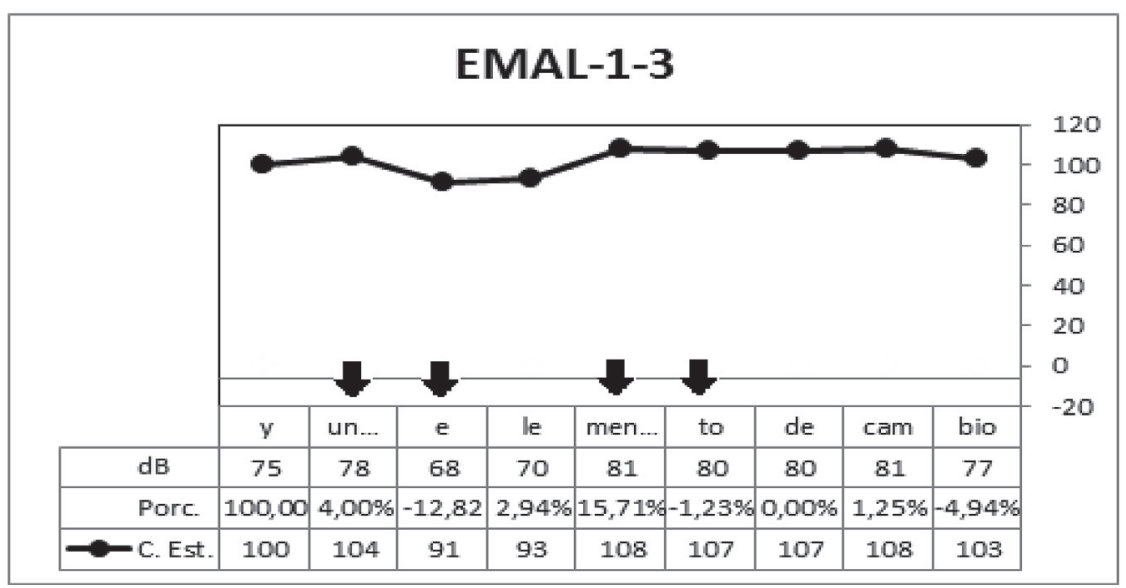

Figura 8. Gráfico de un enunciado malagueño (representación estandarizada de los datos de intensidad).

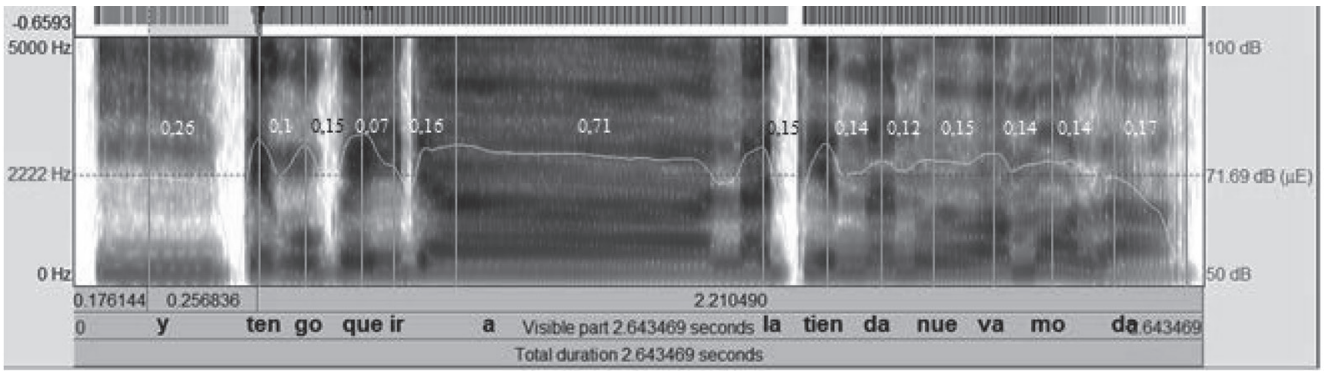

Figura 9. Intervalos de duración (valores absolutos) entre los picos de intensidad de las vocales nucleares en el enunciado madrileño $y$ tengo que ir a la Tienda Nueva Moda.

sería que es más fácil establecer estos puntos que buscar las fronteras silábicas exactas para medir la duración absoluta de cada sílaba. Según Cantero (comunicación personal), no es fácil establecer obviamente las fronteras silábicas en español. Primero, en el español hablado espontáneo, las consonantes en posición posnuclear al final de sílaba suelen ser resilabificadas a la parte prenuclear de la siguiente sílaba si esta comienza por vocal. En decir el, la silabificación más probable sería [de.Өi rel] en vez de [de.Đir el]. Esta sería la primera dificultad encarada si buscamos fronteras silábicas exactas en español. Relacionado con este problema, algunas consonantes tienden a invadir el dominio de la vocal, casi "infectándola", lo que resultaría en una clara imposibilidad de dividir las palabras en sílabas exactamente. Es este el caso, por ejemplo, cuando tenemos [r] en posición implosiva, y si se suprime este sonido por manipulación, la vocal de la sílaba siguiente seguirá percibiéndose rotacizada (Cantero y Ortiz-de-Pinedo Sánchez 2017). Por esta razón se prefiere en este estudio el análisis basado en distancias entre picos de intensidad, ya que tales picos son más fácil y exactamente localizables acústicamente que las fronteras silábicas para medir la duración absoluta de las sílabas. 


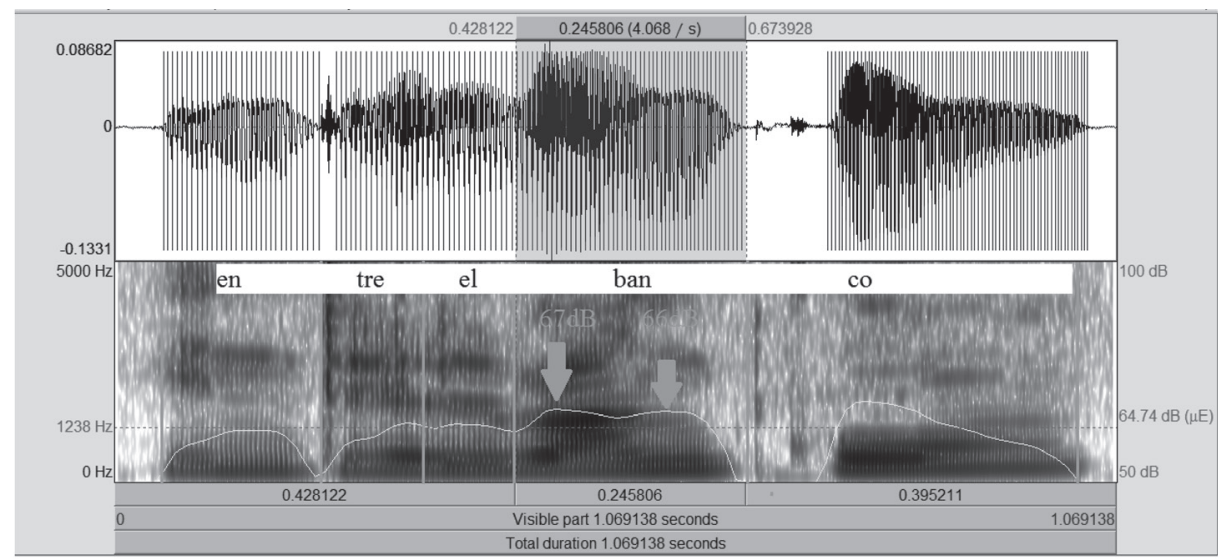

Figura 10. Picos de intensidad vocálico y consonántico dentro de la misma sílaba en un enunciado madrileño, entre el banco.

Para calcular bien los intervalos de duración en este modelo, hay algunos aspectos que se deberían tener en cuenta, para que el análisis no dé resultados falsos. Uno de los aspectos se relaciona con la existencia de varios picos de intensidad dentro de la misma sílaba. En el caso de que haya picos vocálicos y consonánticos en el enunciado, solo debemos representar los valores de los picos vocálicos. Para ejemplificar el problema, véase la Figura 10. En la frase Entre el banco, de un informante madrileño, se ve que existen dos picos de intensidad en la sílaba ban-, pero solo uno de ellos es vocálico, por lo tanto en la medición solo deberíamos tener este pico en cuenta.

Se puede dar, en ciertos casos, el fenómeno de la existencia de más de un pico vocálico dentro de la misma sílaba, como en el caso del enunciado de Badajoz y que el río (Figura 11). En este caso, se debe analizar el pico más alto.

En algunos casos excepcionales, el pico de intensidad no se sitúa en el medio de la vocal, lo que supone un problema para el sistema de estandarización, ya que así no predice bien la duración relativa de la sílaba. Veamos un ejemplo: en el siguiente enunciado, tenemos dos picos en las dos últimas sílabas (-mien- y -to), de 71 y 75 decibelios, respectivamente. El primer pico, de -mien-, no se sitúa en el medio de la vocal nuclear $e$, sino antes, y el pico de la sílaba -to tampoco se sitúa en el medio de la vocal nuclear de - to, sino hacia el final de la sílaba. Esta anormalidad causa que la distancia entre los picos de intensidad en el caso de -mien- sea de 0,38s y la distancia desde el pico de - to hasta el final de la señal acústica (ya que es final del enunciado) sea de 0,15s (Figura 12).

Después de la estandarización, se notaría que el valor de duración relativo correspondiente a la última sílaba -to sería -60,53 (60,53\% menos que el de la sílaba anterior -mien-). Eso, sin embargo, no puede ser el caso, ya que, si examinamos la duración absoluta de estas dos sílabas, y no las distancias entre dos picos de intensidad, -mien- tiene la duración absoluta de 0,29s, en comparación con la duración de la siguiente sílaba -to, de $0,34 \mathrm{~s}$ (Figura 13). El valor de duración relativo en este caso sería $17,24 \%$, un valor positivo en vez del negativo calculado por el análisis PAS. Esto quiere decir que, si los picos de intensidad no se sitúan en el medio de las vocales nucleares, el modelo PAS podría predecir datos de duración incorrectos. 


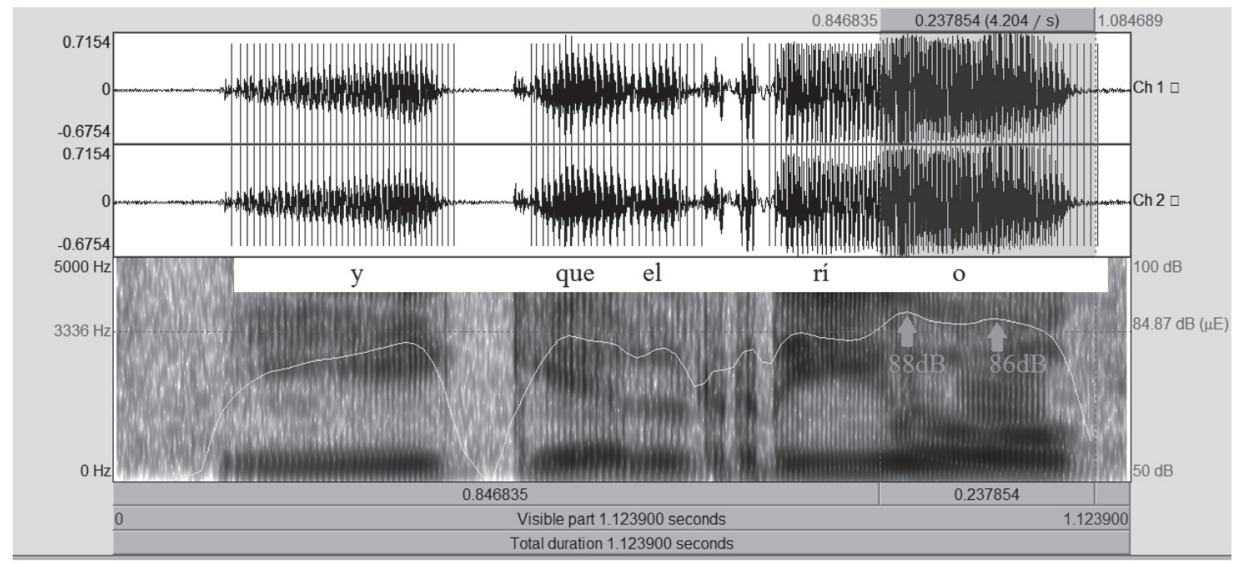

Figura 11. Dos picos vocálicos dentro de la misma sílaba en el enunciado de Badajoz 'y que el río'.

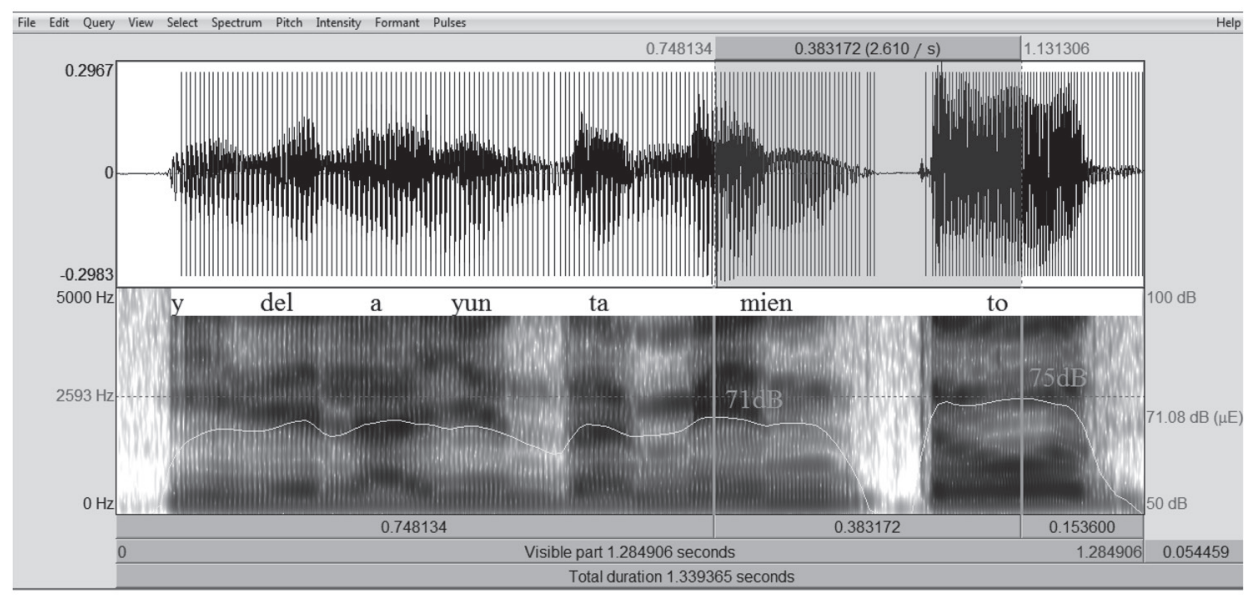

Figura 12. Picos de intensidad no colocados en el medio de las vocales nucleares en las dos últimas sílabas del enunciado madrileño $y$ del ayuntamiento: valores de duración absolutos de las distancias entre picos de intensidad en las dos sílabas. 


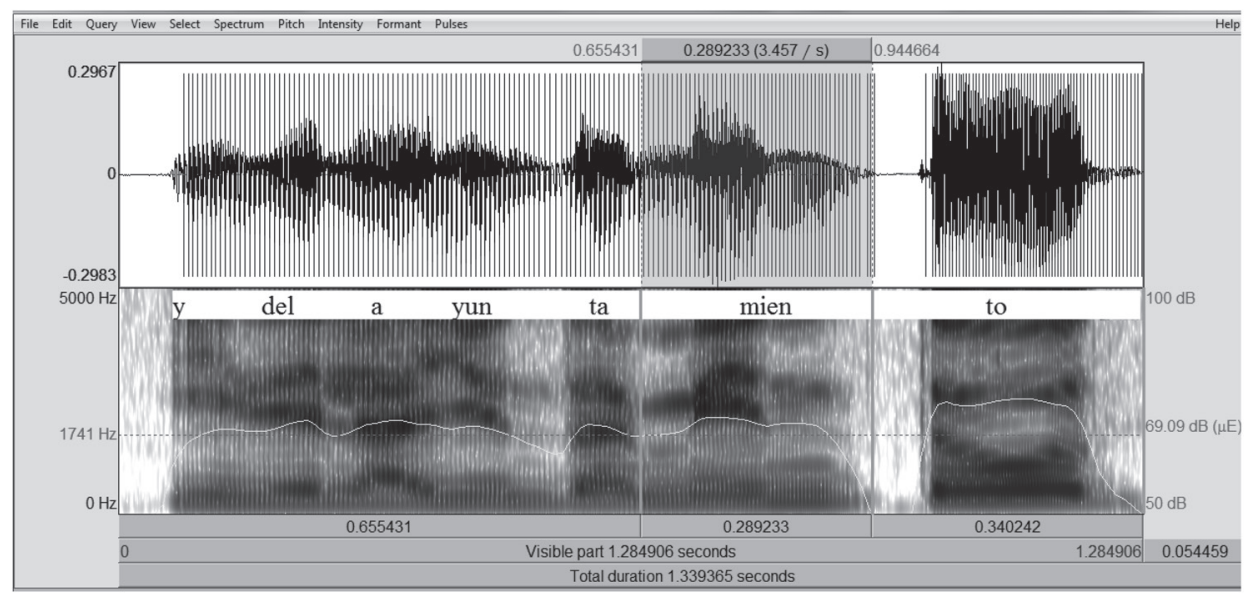

Figura 13. Picos de intensidad no colocados en el medio de las vocales nucleares en las dos últimas sílabas del enunciado madrileño $y$ del ayuntamiento: valores absolutos de la duración de ambas sílabas.

Un problema más surge en casos cuando tenemos dos sílabas adyacentes muy cortas, aun cuando la duración de la segunda sílaba sea el doble de la duración de la primera, pero que sigue siendo muy corta (Figura 14).

En estos casos, el modelo predice que hay un alargamiento del 50\% entre las dos sílabas, pero en realidad esta distancia en segundos es muy corta, apenas perceptible. Por eso nosotros, en este estudio, hemos complementado el modelo PAS y no solo medimos las distancias entre picos de intensidad, sino también, a modo de control, medimos la duración absoluta de las sílabas. Consideramos alargamientos los segmentos con un valor relativo de duración del $+50 \%$ respecto a la sílaba anterior como mínimo, y con una duración absoluta mínima de 0,2s. Este último umbral lo aplicamos, ya que según Goldman-Eisler 1973 y Guaitella 1996, citados por Bondet 2001: 8, la duración mínima de las pausas llenas es de 0,2s y entre la duración de los alargamientos y de las pausas llenas no encontramos diferencias considerables (Machuca et al. 2015). No incluimos en el análisis los alargamientos finales de enunciado, ya que en esta posición la prolongación es un fenómeno natural y no necesariamente intencionado (Gósy y Krepsz 2018).

En nuestro análisis de la duración de las prolongaciones y de las pausas sonoras, hemos recogido dos datos (indicados por flechas en la Figura 15): la duración relativa del segmento afectado (el dato estandarizado, considerando solo los valores superiores a 50\%) y también los valores absolutos. Como solo se consideraron los segmentos con una duración superior a 0,2 segundos, en la Figura. solo se eligieron los datos de un... y men..., ya que en el caso de la sílaba de, aunque la duración del intervalo es del $87,5 \%$ respecto a la duración del intervalo anterior, la duración absoluta del segmento es inferior a 0,2 segundos. En la Figura 15 mostramos cómo se representa esta información en un gráfico de barras:

En cuanto a los valores de duración absoluta, la anotación se realizó basándonos en la información extraída por el software de análisis acústico Praat, teniendo en cuenta el siguiente principio: al medir la duración de las prolongaciones, la sílaba siempre se tomó como el dominio básico del análisis. Esto fue así en el caso del típico fenómeno español de resilabificación (es decir, cuando 


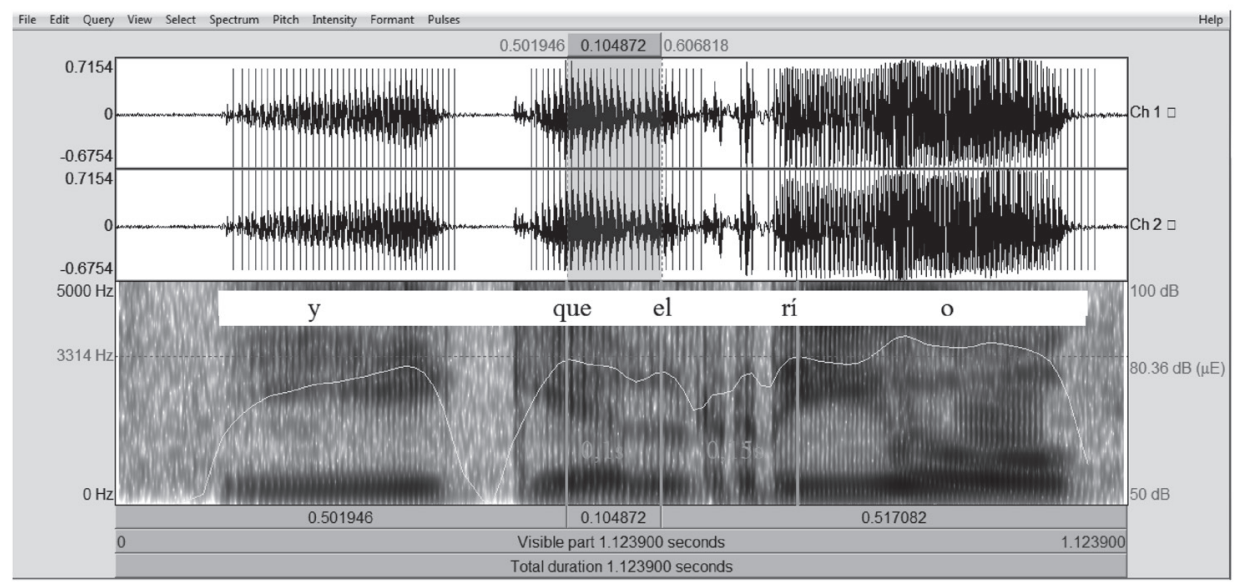

Figura 14. Dos intervalos seguidos de corta duración en el enunciado de Badajoz, y que el río.

EMAL-1-3

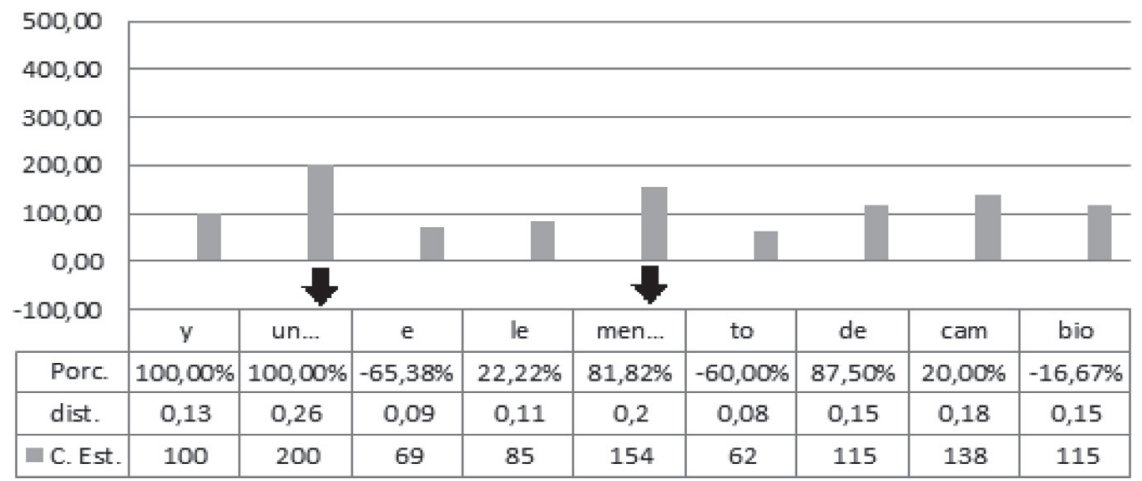

Figura 15. Gráfico de un enunciado malagueño (representación estandarizada de los datos de duración).

una consonante final de palabra se resilabifica como el comienzo de la siguiente palabra si esta comienza con una vocal), como en ves un [be: sun], la duración de la sílaba solo se midió para [be:], ya que la -s se resilabificó a la sílaba siguiente.

\section{Resultados}

A continuación presentaremos los resultados obtenidos en los tres aspectos analizados, la melodía, la intensidad y la duración de las hesitaciones, en una tabla (Tabla 2). Después, analizaremos brevemente los tres aspectos investigados separadamente. 


\begin{tabular}{|c|c|c|c|}
\hline \multicolumn{2}{|c|}{ Características prosódicas de los alargamientos y pausas llenas (medias) } & Norte & Sur \\
\hline \multicolumn{2}{|l|}{ movim. tonal hasta síl. afectada (\%) } & 0,88 & 1,75 \\
\hline resultados de la prueba $t$ (varianzas iguales) & \multicolumn{3}{|c|}{$\mathrm{P}(\mathrm{T}<=\mathrm{t})$ dos colas: 0,$11 ;<\mathrm{t}$ valor crítico: 1,97} \\
\hline \multicolumn{2}{|l|}{ movim. tonal desde síl. afectada (\%) } & $-0,16$ & $-4,15$ \\
\hline resultados de la prueba t (varianzas iguales) & \multicolumn{3}{|c|}{$\mathrm{P}(\mathrm{T}<=\mathrm{t})$ dos colas: 0,$77 ;<\mathrm{t}$ valor crítico: 1,97} \\
\hline \multicolumn{2}{|l|}{ movim. dinámico hasta síl. afectada (\%) } & 2,22 & 1,22 \\
\hline resultados de la prueba $\mathrm{t}$ (varianzas iguales) & \multicolumn{3}{|c|}{$\mathrm{P}(\mathrm{T}<=\mathrm{t})$ dos colas: 0,$26 ;<\mathrm{t}$ valor crítico: 1,97} \\
\hline \multicolumn{2}{|l|}{ movim. dinámico hasta síl. afectada (\%) } & $-1,08$ & $-1,46$ \\
\hline resultados de la prueba t (varianzas desiguales) & \multicolumn{3}{|c|}{$\mathrm{P}(\mathrm{T}<=\mathrm{t})$ dos colas: 0,$73 ;<\mathrm{t}$ valor crítico: 1,97} \\
\hline \multicolumn{2}{|l|}{ distancia entre picos de intensidad (\%) } & 164,4 & 166,9 \\
\hline \multicolumn{2}{|c|}{ Características prosódicas de los alargamientos y pausas llenas (medias) } & Norte & Sur \\
\hline resultados de la prueba t (varianzas iguales) & \multicolumn{3}{|c|}{$\mathrm{P}(\mathrm{T}<=\mathrm{t})$ dos colas: 0,$88 ;<\mathrm{t}$ valor crítico: 1,97} \\
\hline \multicolumn{2}{|l|}{ duración absoluta del segmento (s) } & 0,44 & 0,4 \\
\hline resultados de la prueba t (varianzas desiguales) & \multicolumn{3}{|c|}{$\mathrm{P}(\mathrm{T}<=\mathrm{t})$ dos colas: 0,$15 ;<\mathrm{t}$ valor crítico: 1,97} \\
\hline
\end{tabular}

Tabla 2. Características prosódicas de los alargamientos y pausas llenas.

\subsection{El aspecto melódico de las hesitaciones en ambos corpus}

El valor medio de la proporción del movimiento tonal hasta la sílaba afectada, en el caso de los dialectos norteños, es del $0,88 \%$, mientras que, en el caso de los sureños, es del 1,75\%. Los valores medios desde la sílaba afectada son del $-0,16 \%$ en el caso de los dialectos septentrionales y - $4,15 \%$ en el caso de los meridionales (véase Figura 16).

Según los resultados de la prueba t, la diferencia no es significativa en el intervalo de confianza del 95\% entre las medias en ninguno de los dos casos.

\subsection{El aspecto dinámico de las hesitaciones en ambos corpus}

Se desprende de los datos que, en los dialectos septentrionales, el valor medio de la proporción del cambio dinámico hasta la sílaba afectada es del 2,22\%, mientras que, en los meridionales, el valor medio es del 1,22\%. Los valores desde la sílaba afectada tampoco presentan diferencias considerables, siendo el valor medio del corpus septentrional -1,08\%, mientras que el del sur, ligeramente menos, $-1,46 \%$ (Figura 17).

Según la prueba t de dos muestras, no hay una diferencia significativa entre las medias en ninguno de los dos casos en el intervalo de confianza del $95 \%$. 


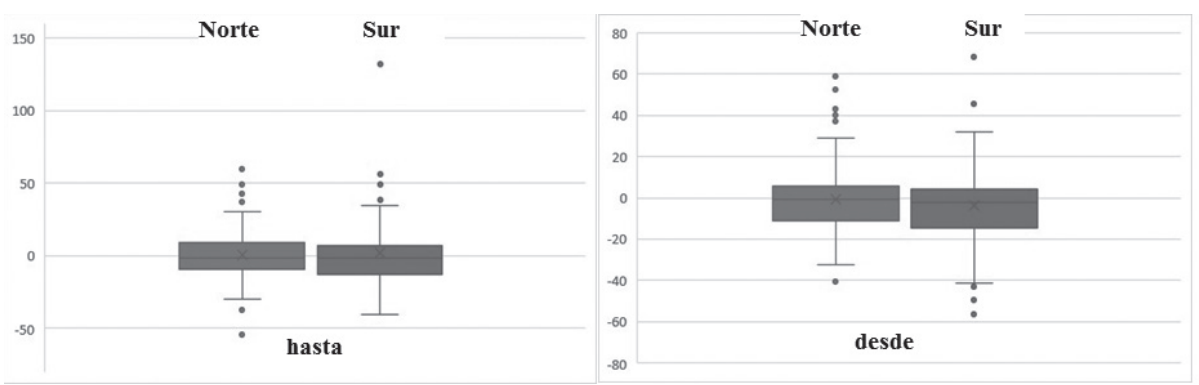

Figura 16. Proporción del movimiento tonal hasta y desde la sílaba afectada (\%) (los dos corpus, alargamientos y pausas sonoras).

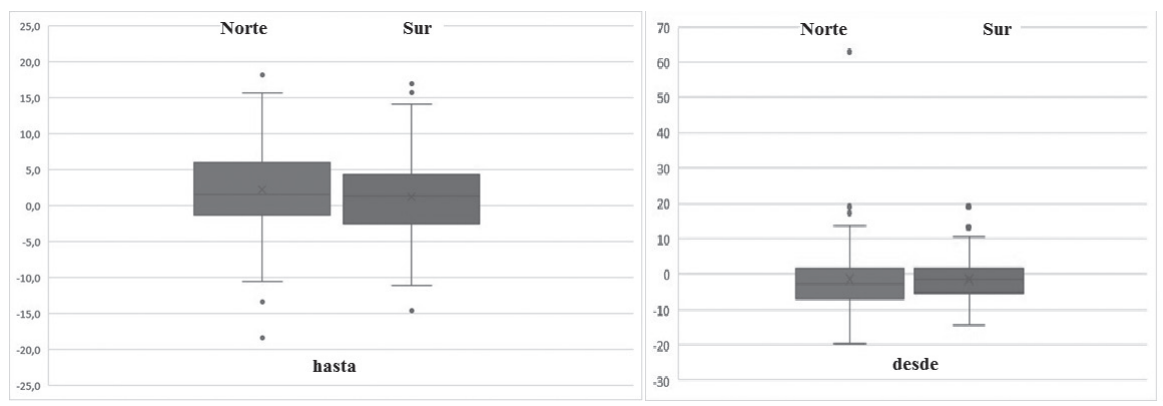

Figura 17. Intensidad: valores hasta y desde la sílaba afectada (\%) (los dos corpus, alargamientos y pausas sonoras).

\subsection{El aspecto rítmico de las hesitaciones en ambos corpus}

En términos absolutos, no encontramos diferencia considerable entre los valores medios $(0,44 \mathrm{~s}$ en el caso de los dialectos septentrionales contra el valor medio de $0,4 \mathrm{~s}$ en el caso de los meridionales). Los valores de duración relativa en los dos corpus también son casi idénticas (164,4\% y $166,9 \%$, respectivamente, véase Fig. 18).

Según la prueba t de dos muestras, la diferencia de estas medias tampoco resultó ser estadísticamente significativa en el intervalo de confianza del 95\%. 


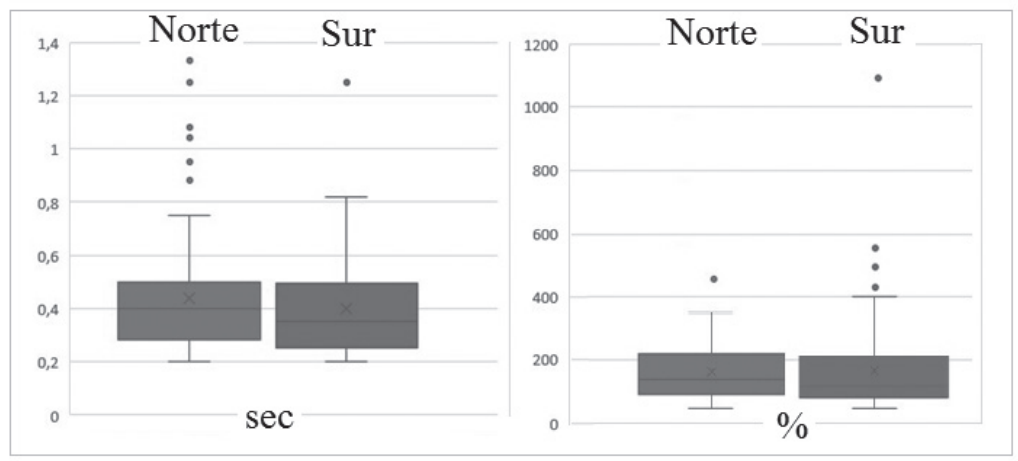

Figura 18. Duración: valores absolutos de segmentos (s) y valores medios de distancia tonal entre picos (\%) (los dos corpus, alargamientos y pausas sonoras).

\section{Conclusiones y discusión}

En el presente estudio, analizamos dos fenómenos de hesitación: las pausas llenas y los alargamientos en los dialectos septentrionales y meridionales del español desde el punto de vista de tres aspectos prosódicos: la melodía, la intensidad y la duración. Nos hemos valido de 200 enunciados espontáneos, 100 de cada una de las dos zonas dialectales, y utilizamos el protocolo de análisis prosódico trifásico conceptuado por Cantero (2019). Hemos formulado dos hipótesis relacionadas con la prosodia de las hesitaciones españolas investigadas en los dos dialectos:

1) en cuanto a las pausas llenas y las prolongaciones, no habrá diferencias relevantes en los valores relativos de la melodía y de la intensidad, pero sí en el aspecto de la duración. La hipótesis se basa en la suposición de que, en los dialectos meridionales, que son más rápidos por la frecuente elisión (Toledo 2010), la duración relativa de los segmentos alargados y las pausas llenas respecto a los segmentos anteriores es más larga que en los dialectos norteños.

2) los segmentos alargados y las pausas llenas que carecen de función comunicativa específica y solo sirven para mantener el turno de palabra del hablante no se caracterizarán por movimientos tonales y dinámicos llamativos respecto a su contexto. Como su función es garantizar que el hablante no pierda el turno de palabra, esto solo se logra, supuestamente, si los segmentos afectados no entrecortan el discurso con una melodía o intensidad saliente.

Respecto a nuestra hipótesis (1), según nuestros resultados, vemos que, en el caso de los dos dialectos, no encontramos diferencias considerables en los siguientes dos aspectos: la proporción de movimientos en y desde el segmento prolongado afectado y las pausas llenas en cuanto a la melodía y a la intensidad, tal como lo hemos predicho. Sin embargo, al contrario de nuestra hipótesis, tampoco existen diferencias relevantes en el aspecto de la duración.

Nuestra hipótesis (2) parece estar confirmada, ya que los movimientos tonales y dinámicos asociados a los alargamientos y a las pausas llenas no son de una proporción llamativa (los valores 
medios no alcanzan en los aspectos investigados ni el 5\%). En cuanto a las pausas llenas, en este aspecto coincidimos con las observaciones de Garrido et al. (2017) y Gil et al. (2018), según las cuales las pausas llenas sin función comunicativa específica se caracterizan por un tono aplanado o ligeramente descendente en el español. La predominancia de contornos planos y descendentes en las pausas llenas también parece ser típico del francés (Duez 2001) y otros idiomas (Giannini 2003, Vasilescu y Adda-Decker 2007); Candea et al. (2005) encontraron este contorno aplanado característico de las pausas llenas en ocho lenguas (incluyendo el español latinoamericano), lo que los llevó a suponer que se trataba de un rasgo universal. Ya sabemos que, en el caso de la entonación española, el umbral de percepción es del 10\% como mínimo (Font-Rotchés y Mateo Ruiz 2011). Serían necesarias pruebas perceptivas de la misma índole en el caso de la intensidad también para ver si efectivamente estos valores de intensidad también deberían considerarse bajos, o ya son relevantes.

El tema de medir la duración relativa de los segmentos prolongados plantea varias cuestiones de análisis. La primera se relaciona con la necesidad de establecer un umbral de percepción para las prolongaciones: desde qué porcentaje de alargamiento se debería considerar un segmento hasta ya un segmento prolongado. Los valores mínimos de un umbral de percepción en el caso de los valores relativos serían necesarios, ya que nuestros valores mínimos utilizados en este estudio son arbitrarios (un alargamiento del 50\% como mínimo). Según el método utilizado por Deme y Markó (2013), un segmento fue considerado prolongado en el caso de que 6 de 10 lingüistas consideraran que el segmento en cuestión estaba prolongado, pero no habían establecido ningún valor mínimo como umbral de percepción. En lo referente a los valores absolutos, nos hemos valido del umbral de 0,2 segundos (valor mínimo habitual en la literatura, véase Gil et al. 2018), pero ciertos autores (como Blondet 2006, citado por Rodrígez et al. 2015), por ejemplo, lo definen a partir de 0,5 segundos, mientras que Hieke et al. 1983 (citados por Campione y Véronis 2005) y Candea et al. 2005 permiten valores incluso inferiores a 0,2s, por lo tanto no existe unanimidad respecto a este valor mínimo.

También se debe plantear la cuestión de si la mente humana identifica un segmento alargado respecto a su contexto adyacente, o se trata de una duración media almacenada mentalmente, respecto a la cual cada segmento nuevo se mide y se compara. De ser así, el modelo PAS no sería suficiente para describir las duraciones relativas.

\section{Referencias bibliográficas}

Adriaens, L. M. H. (1991). Ein Modell deutscher Intonation. Eine experimentell-phonetische Untersuchung nach den perzeptiv relevanten Grundfrequenzänderungen in vorgelesenem Text. $\mathrm{PhD}$ thesis, Technological University of Eindhoven.

Baditzné Pálvölgyi, K. (2020). Hesitation patterns in Northern and Southern dialects of European Spanish. Presentation at the 17th Old World Conference on Phonology, 5-7th of February, 2020. University of Warsaw. . (2012). Spanish Intonation of Hungarian Learners of Spanish: yes-or no questions. Tesis Doctoral. Budapest: Eötvös Loránd University. Biblioteca Phonica 15 (2012), <http://www.publicacions.ub.edu/revistes/phonica-biblioteca/> 
Beaugendre, F. (1994). Une étude perceptive de l'intonation du français. PhD Thesis, University of Paris XI. Blondet, M. A. (2001). Las pausas llenas: marcas de duda e identidad lingüística. Lingua Americana, 5, 8, 5-15. Blondet, M. (2006). Variaciones de la velocidad de habla en español: patrones fonéticos y estrategias fonológicas. Un estudio desde la producción. Tesis doctoral. Mérida: Universidad de Los Andes.

Boersma, P.; \& Weenink, D. (2019). Praat: Doing phonetics by computer (version 6.0.49). <https://www.fon. hum.uva.nl/praat/>

Campione, E.; \& Véronis, J. (2005). Pauses and hesitations in French spontaneous speech. DiSS'05, Disfluency in Spontaneous Speech Workshop, 43-46.

Candea, M.; Vasilescu, I.; \& Adda-Decker, M. (2005). Inter- and intra-language acoustic analysis of autonomous fillers. DISS' 05, Disfluency in Spontaneous Speech Workshop, 47-52.

Cantero Serena, F. J. (2019). Análisis prosódico del habla: más allá de la melodía. In M. R. Álvarez Silva, A. Muñoz Alvarado, \& L. Ruiz Miyares (Eds.), Comunicación Social: Lingüística, Medios Masivos, Arte, Etnología, Folclor y otras ciencias afines. Volumen II (pp. 485-498). Santiago de Cuba: Ediciones Centro de Lingüística Aplicada.

Cantero Serena, F. J.; Alfonso, R.; Bartolí, M., Corrales, A.; \& Vidal, M. (2005). Rasgos melódicos de énfasis en español. Laboratori de Fonètica Aplicada - LFA. Phonica, 1., <http://www.publicacions.ub.edu/revistes/phonica1/PDF/articulo_03.pdf>

Cantero, Francisco J.; \& Font-Rotchés, D. (2020). Melodic Analysis of Speech (MAS). Phonetics of Intonation. In J. Abasolo, I. de Pablo, \& A. Ensunza (Eds), Contributions on education (pp. 20-47). Universidad del País Vasco.

de dispersion. Moenia, 13, 69-92.

- (2009). Protocolo para el análisis melódico del habla, Estudios de Fonética Experimental, 18, 17-32.

Cantero Serena, F. J.; \& Ortiz-de-Pinedo Sánchez, N. (2017). La vocal rotizada o r-coloring en el español peninsular. In V. Marrero Aguiar, \& E. Estebas Vilaplana (Eds.): Tendencias actuales en fonética experimental: Cruce de disciplinas en el centenario del Manual de Pronunciación Española (Tomás Navarro Tomás) (pp. 49-52). Madrid: UNED.

de Leeuw, E. (2007). Hesitation markers in English, German, and Dutch. Journal of Germanic Linguistics, 19, $2,85-114$.

Deme, A.; \& Markó, A. (2013). Lengthenings and filled pauses in Hungarian adults' and children's speech. In R. Eklund (Ed.), Proceedings of DiSS 2013, The 6th Workshop on Disfluency in Spontaneous Speech KTH Royal Institute of Technology (pp. 21-24). Stockholm: Department of Speech Communication and Music Acoustics, Royal Institute of Technology.

Duez, D. (2001). Caractéristiques acoustiques et phonétiques des pauses remplies dans la conversation en français. Travaux Interdisciplinaires du Laboratoire Parole et Langage, 20, 31-48.

Eklund, R. (2004). Disfluency in Swedish human-human and human-machine travel booking dialogues. PhD thesis, Linköping Studies in Science and Technology, Dissertation No. 882, Department of Computer and Information Science, Linköping University, Sweden.

Estruch, M.; Garrido, J. M.; Llisterri, J.; \& Riera, M. (2007). Técnicas y procedimientos para la representación de las curvas melódicas. RLA, Revista de Lingüística Teórica y Aplicada 45, 59-87. <http://liceu.uab.es/ joaquim/publicacions/Estruch_Garrido_Llisterri_Riera_Metodos_Entonacion_07.pdf>

Font-Rotchés, D. (2007). L'entonació del català. Biblioteca Milà i Fontanals 53. Barcelona: Publicacions de l'Abadia de Montserrat. 
tura, 19, 299-329.

. (2009). Les interrogatives pronominals del català central. Anàlisi melòdica i patrons entonatius. Els Marges. Revista de llengua i literatura, 87, 41-64.

Font-Rotchés, D.; \& Mateo Ruiz, M. (2011). Absolute interrogatives in Spanish: a new melodic pattern. Actas do VII congresso internacional da ABRALIN (pp. 1111-1125). Curitiba (Brasil).

Garrido Almiñana, J. M.; Laplaza, Y.; García, C. L. (2017). La caracterización pragmática y prosódica de la vocalización "mmm" en español. In V. Marrero Aguiar, \& E. Estebas Vilaplana (Eds.): Tendencias actuales en fonética experimental: Cruce de disciplinas en el centenario del Manual de Pronunciación Española (Tomás Navarro Tomás) (pp. 125-129). Madrid: UNED.

Garrido Almiñana, J. M. (1991). Modelización de patrones melódicos del español para la síntesis y el reconocimiento. Bellaterra: Departament de Filologia Espanyola, Universitat Autònoma de Barcelona.

- (1996). Modelling Spanish Intonation for Text-to-Speech Applications. PhD dissertation. Departament de Filologia Espanyola, Universitat Autònoma de Barcelona.

Giannini, A. (2003). Hesitation phenomena in spontaneous Italian. Proc. of the XV ICPhS, 2653-2656.

Gil Fernández, J.; Lahoz-Bengoechea, J. M.; \& Villa, J. (2018). La vocal de relleno en español y en ruso: caracterización acústica e implicaciones teóricas. Estudios Filológicos (pp. 69-94). Santiago: Universidad de Chile.

Goldman-Eisler, F. (1973). Psycholinguistics. Experiments in Spontaneous Speech. New York: Academic Press.

Gósy, M. (2002). A megakadásjelenségek eredete a spontán beszéd tervezési folyamatában [El origen de los fenómenos de disfluencia en el proceso de la planificación del habla]. Magyar Nyelvőr, 126, 192-204.

Gósy, M.; \& Krepsz, V. (2018). Phrase-final Lengthening of Phonemically Short and Long Vowels in Hungarian Speech across Ages. In M. Gósy, \& T. E. Gráczi, T. E. (Eds.) Challenges in analysis and processing of spontaneous speech (pp. 99-126). Research Institute for Linguistics, Hungarian Academy of Sciences.

Guaitella, I. (1996). Analyse prosodique des hésitations vocales: propositions pour un modèle rythmique. R.P.A., 118-119, 113-145.

Hieke, A.; Kowal, S.; \& O’Connell, D. C. 1983. The trouble with “articulatory" pauses. Language and Speech, 26, 203-214.

Hualde, J. I. (2014). Los sonidos del español. Cambridge: Cambridge University Press.

Kao, W. (2011). La entonación de enunciados declarativos e interrogativos en chino mandarín hablado por taiwaneses. Trabajo de investigación final de Máster. Laboratori de Fonètica Aplicada de la UB.

Lickley, R. J. (1994). Detecting disfluency in spontaneous speech. PhD dissertation, University of Edinburgh.

. (2015). Fluency and disfluency. In M. Redford (Ed.), The handbook of Speech production (pp. 445-469). Malden: Wiley-Blackwell.

Maclay, H.; \& Osgood, C. (1959). Hesitation phenomena in spontaneous English speech. Word, 15, 19-44.

Machuca Ayuso, M. J. (2018). Pausas sonoras y bilingüismo. In Estudios de Fonética Experimental, XXVII, 75-95.

Machuca, M. J.; Llisterri, J.; \& Ríos, A. (2015). Las pausas sonoras y los alargamientos en español: un estudio preliminar. Normas. Revista de Estudios Lingüísticos Hispánicos, 5, 81-96.

Neuberger, T. (2014). A spontán beszéd sajátosságai gyermekkorban. [Las características del habla espontánea en la niñez]. Beszéd. Kutatás. Alkalmazás. Budapest: ELTE Eötvös Kiadó. 
Odé, C.; \& van Heuven, V. J. (1994). Experimental studies of Indonesian prosody. Dep. of Languages and Cultures of Southeast Asia and Oceania, University of Leiden.

Olaszy, G.; \& Koutny, I. (2001). Intonation of Hungarian Questions and their prediction from text. In S. Puppel, \& G. Demenko (Eds.), Prosody 2000, Speech recognition and synthesis, Poznań 2001.

Patiño, E. (2008). Prosodic Comparative Study of Mexico City and Madrid Spanish. Freie Universität Berlin, Germany. Escuela Nacional de Antropología e Historia, México.

Prieto, P.; Borràs-Comes, J.; \& Roseano, P. (Coords.) (2010-2014). Interactive Atlas of Romance Intonation. $<$ http://prosodia.upf.edu/iari/>

Rebollo Couto, L. (1997). Pausas y ritmo en la lengua oral. Didáctica de la pronunciación. In ASELE Actas VIII (pp. 667-676). Centro Virtual Cervantes.

Rodríguez, L. J. et al. (2001). Annotation and analysis of disfluencies in a spontaneous speech corpus in Spanish. In ITRW on Disfluency in Spontaneous Speech (Diss '01) (pp. 1-4). Edinburgh, Scotland, UK, August 29-31.

. (2015). Las pausas en el discurso de individuos con demencia tipo Alzheimer. Estudio de casos. Revista de Investigación en Logopedia, 1, 40-59.

Shriberg, E. (1994). Preliminaries to the theory of speech disfluencies. PhD dissertation, University of California, Berkeley.

Stepanova, S. (2007). Some features of filled hesitation pauses in spontaneous Russian. In J. Trouvain, \& W. J. Barry (Eds.): Proceedings of ICPhS 2007. 16th International Congress of Phonetic Sciences, Saarbrücken, pp. 1325-1328.

T’Hart, J. T.; Collier, R.; \& Cohen, A. (1990). A perceptual study of intonation. An experimental-phonetic approach to speech melody. Cambridge: Cambridge University Press.

Toledo, G. (2010). Métricas rítmicas en tres dialectos Amper-España. Estudios Filológicos, 45, 93-110.

Vasilescu, I.; \& Adda-Decker, M. (2007). A cross-language study of acoustic and prosodic characteristics of vocalic hesitations. In A. Esposito, M. Bratanic, E. Keller, M. Marinaro (Eds.), Fundamentals of Verbal and Nonverbal Communication and the Biometric Issue (pp. 140-148), Amsterdam: IOS Press.

Villa Villa, J. et al. (2017). Las vocales de relleno en español: nuevos datos y algunas reflexiones. In Ruiz Minares et al. (Eds), Nuevos estudios sobre Comunicación Social, Vol. I. (pp. 165-169). Centro de Linguistica Aplicada, Santiago de Cuba.

\section{Fuentes electrónicas:}

Fuente de la imagen de la Figura 1: <https://www.freepik.com/free-vector/map-spain_2454242.htm\#page $=1 \&$ query $=$ spain $\% 20$ maps $\&$ position $=4>$

Fuentes de las entrevistas [última consulta 12/02/2020]:

De Cerca - Entrevista a Miguel Ángel Heredia, número 1 del PSOE Málaga al Congreso (2016) https://www.youtube.com/watch?v=T1_-NEVxenY

El alcalde de Salamanca, Carlos García Carbayo, en Hoy por hoy (2019) https://www.youtube.com/watch?v=M7DE3lRDF94

Elías Bendodo y Patricia del Pozo visitan el Museo de Málaga (2019) https://www.youtube.com/watch?v=wvOgYVpfnuE 
Entrevista a fondo a Miguel Ángel García Nieto (2017) https://www.youtube.com/watch?v=sXM3jXRps2s

Entrevista a Javier Iglesias presidente de la diputación (2018) https://www.youtube.com/watch?v=FrogFcXfguw

Entrevista a Juan Vicente Herrera Candidato PP Junta Castilla y León (2015) https://www.youtube.com/watch?v=JJSG2A_GWk8

Entrevista a Luis Tudanca Candidato PSOE Junta de Castilla y León (2015) https://www.youtube.com/watch?v=9123pazzakY

Entrevista con Antonio Silván, Alcalde de León (2018) https://www.youtube.com/watch?v=4qcSy7tUQmg\&t=21s

Entrevista con José Antonio Díez, Alcalde de León (2019) https://www.youtube.com/watch?v=BsNrr-cHPTw\&t=87s

Francisco Javier Fragoso, Alcalde de Badajoz en FITUR (2018) https://www.youtube.com/watch?v=3ENRGwVlV5o\&t=221s

Guillermo Fernández Vara: "Sánchez sabía que le iban a partir la cara desde el minuto uno", (2018) https://www.youtube.com/watch?v=X8gs7EIh2_0

Interrogatorio de Gabriel Rufián a Ángel Acebes por la corrupción del PP (2018) https://www.youtube.com/watch?v=L3mJSvblKt8\&t=544s

La Entrevista | Juan Espadas, alcalde de Sevilla (2018) https://www.youtube.com/watch?v=a8Mq7eRms7M

Luisa García Chamorro en Canal Sur, (2015) https://www.youtube.com/watch?v=q2p89F0Cjvo

María José López González (versión extendida) (2015) https://www.youtube.com/watch?v=7G1LW1nsCcw 
